\title{
Geographie und Infrastruktur
}

\section{1. Übersicht}

Die Infrastruktur hat in der Systematik der neueren allgemeinen Anthropogeographie in Deutschland noch keinen festen Platz eingenommen. Sehen wir das - in der Regel sehr differenzierte - Inhaltsverzeichnis der verschiedenen Lehrbücher zur Wirtschaftsgeographie (OTREMBA 1960, VOPPEL 1970 und BOESCH 1977) durch, fehlt der Begriff der Infrastruktur völlig. Auch in der «Bibliographie zum Geographiestudium» von BLOTEVOGEL/HEINEBERG (1976) fehlt in der tiefgegliederten Systematik ein Hinweis auf die Infrastruktur. Allein in der "Wirtschaftsgeographie» von SCHÄTZL (1978) gibt es ein Unterkapitel «Infrastruktur». Ursache für die Berücksichtigung der Infrastruktur wird weniger das junge Erscheinungsjahr dieses Bandes sein als vielmehr die Tatsache, daß SCHÄTZL von den Wirtschaftswissenschaften her zur Geographie gekommen ist und in seinem Lehrbuch einen raumwirtschaftlichen Ansatz verfolgt. Gewiß nicht zufällig sind alle grundlegenden und umfassenden Beiträge zur Theorie der Infrastruktur in keinem Fall von einem Geographen verfaßt worden (JOCHIMSEN 1966, JANSEN 1968, FREY 1972, BUHR 1975). Ist die Infrastruktur also kein Forschungsgegenstand der Anthropogeographie?

\section{Definitionen und Zielsetzungen}

Der Begriff der Infrastruktur fand zunächst eine gewisse Verbreitung durch die Fachsprache der NATO, bevor er Ende der 50er und vor allem Anfang der $60 \mathrm{er}$ Jahre in die Wirtschaftswissenschaften und in die Regionalpolitik eingeführt wurde. Als Infrastruktur werden öffentlich nutzbare Güter bezeichnet, deren Erstellung Investitionen erfordert und die eine unerläßliche Voraussetzung für die Wirtschaft darstellen. Sie wird untergliedert in die materielle, institutionelle und personelle Infrastruktur. Dabei liegt das Schwergewicht auf der materiellen Infrastruktur, also auf den Einrichtungen zur Energieund Wasserversorgung, des Verkehrs und der Telekommunikation, des Bildungs- und Gesundheitswesens, für die Verwaltung u.ä.m.

Als wesentliche Ziele der Infrastrukturforschung sieht JOCHIMSEN (1966) das Gewinnen von empirisch gehaltvollen Hypothesen und Theoremen in folgenden Bereichen:

- das Bestimmen des Einflusses der Infrastruktur auf das Niveau der Wirtschaftstätigkeit in einer Region.

- Klären der Frage, inwieweit die Infrastruktur eine Voraussetzung für die sozioökonomische Entwicklung einer Region ist.

Diese Zielsetzungen sind vor allem auf die Wirtschaftsentwicklung von Regionen bezogen. Es werden direkt wirksame Infrastrukturinvestitionen von den indirekt wirksamen unterschieden: Die direkt wirksame Infrastruktur dient den gewerblichen Unternehmen ganz oder überwiegend, während die übrige Infrastruktur zunächst die allgemeinen Bedürfnisse der Bevölkerung befriedigt, dadurch aber auch die Standortbedingungen für die Unternehmen beeinflußt. In der Geographie, aber auch in der Umgangssprache ist diese Differenzierung jetzt weitgehend in den Hintergrund getreten. Wie noch zu zeigen sein wird, richtet sich das geographische Forschungsinteresse primär auf die indirekt wirksame Infrastruktur.

Innerhalb der Geographie befaßt sich teilweise der sozialgeographische, vor allem aber der standorttheoretische Ansatz als geographische Subdisziplin der Sozial- und Wirtschaftswissenschaften mit der Infrastruktur (HARD 1973, 187; entsprechende Fachvertreter sind in Kap. 4 genannt). Gegenstand des standorttheoretischen Ansatzes ist nach HARD (1973, 184 f.) die räumliche Organisation der Gesellschaft, und er befaßt sich damit zumindest partiell mit der Lokalisation der Infrastruktur. Es werden

- Verbreitungs-, Verknüpfungs- und Ausbreitungsmuster quantitativ-statistisch beschrieben und durch entsprechende Beschreibungsmodelle dargestellt,

- Standorte und Standortsysteme nach Lokalisierung, Aktivitäten und Beziehungen erklärt und prognostiziert, sie werden als Ergebnisse von Standortentscheidungen bestimmter Entscheidungsträger interpretiert,

Prof. Dr. Christoph Becker, Geographisches Institut Universität Trier, D-5500 Trier. 
- Standortansprüche und -entscheidungen der Beteiligten rekonstruiert und prognostiziert.

Es wird im folgenden zu prüfen sein, unter welcher Perspektive die empirischen Arbeiten zur Wirtschafts- und Sozialgeographie die verschiedenen Arten der Infrastruktur behandeln und inwieweit sich die Geographie überhaupt an der Erforschung der Infrastruktur beteiligt.

\section{Die Entwicklung in den Betrachtungs- weisen}

Bis weit in die 60er Jahre beschränkte sich die Angewandte Geographie auf die Beschreibung und Erklärung von Strukturen und Entwicklungen. Charakteristisch für diese Phase sind die Planungsatlanten der Länder, in denen u.a. die Verbreitung verschiedener Infrastruktureinrichtungen dargestellt ist. Bei dieser chorologischen Betrachtungsweise entstanden nur gelegentlich Ansätze zur Theoriebildung. Die Verbreitungskarten verbesserten zwar die Informationsbasis in der Raumordnung, Hinweise für die Planung der Infrastruktur wurden jedoch kaum entwickelt.

Im Gefolge der Konjunkturkrise 1966/67 wuchs zunächst bei der Bundesraumordnung - das Bedürfnis, die Effizienz der regionalpolitischen Instrumente zu verbessern. Die Diskussion wurde durch das Ausweisen von "raumwirksamen Maßnahmen» der Bundesregierung im Raumordnungsbericht 1968 stärker in Gang gebracht. Es entwickelte sich eine Diskussion über die raumordnungspolitischen Ziele, und eine Erfolgskontrolle wurde angestrebt, bei der wir heute eine Vollzugs-, eine Zielerreichungs- und eine Wirkungskontrolle unterscheiden.

Diese Erfolgskontrolle bezieht sich auf alle raumwirksamen staatlichen Aktivitäten, unter denen die Infrastruktur wegen ihres im allgemeinen großen raumordnungspolitischen Effektes besondere $\mathrm{Be}$ deutung genießt.

Die Vollzugskontrolle geht der Frage nach, wo, wann und welche Mittel für die verschiedenen Arten der Infrastruktur ausgegeben wurden. Diese monetäre Betrachtungsweise kann eine gute Voraussetzung für weitere Analysen sein, weniger eine Grundlage für planerisches Handeln. Geographische Beiträge dazu liegen von KÜHN (1972), BOESLER (1972), REUTER (1974), ten BRINK/GATZWEILER (1976) und ISTEL (1976) vor; allerdings dominieren in diesem Bereich Arbeiten von Wirtschaftswissenschaftlern.

Mit Ist-Soll-Vergleichen im Rahmen einer Zielerreichungskontrolle haben sich Geographen bislang nicht befaßt. Wirkungskontrollen, bei denen die Effekte bestimmter infrastruktureller Maßnahmen im Rahmen von Fallstudien analysiert werden, wurden von Geographen gelegentlich gezielt durchgeführt, vor allem aber können hier zahlreiche geographische
Arbeiten eingeordnet werden. Obwohl auch bei den Wirkungskontrollen wirtschaftswissenschaftliche Untersuchungen überwiegen, bieten sie ein Arbeitsfeld, für das Geographen günstige Voraussetzungen mitbringen: Sie sind gewohnt, viele Aspekte zu integrieren und besonders auch die räumliche Perspektive zu beachten, so daß sie die Vielfalt der Auswirkungen im Raum erkennen, erfassen und gewichten können. Eine theoretische Übersicht über die «Auswirkungen von Infrastrukturinvestitionen» bieten HANSER et al. (1979).

\section{Geographische Arbeiten in verschiedenen Infrastrukturbereichen}

In der Anthropogeographie hat sich der Schwerpunkt der Forschungsarbeiten zur Sozialgeographie hin verlagert. Wenn dabei vor allem das sozialgruppenspezifische Verhalten bei der Ausübung der Daseinsgrundfunktionen untersucht wird, kann dies in der Regel nur mit engem Bezug zur Infrastruktur geschehen, da sie den materiellen Rahmen für die Ausübung der Daseinsgrundfunktionen absteckt. Allerdings gilt in der Sozialgeographie das primäre Interesse dem Verhalten menschlicher Individuen im Raum, während die Infrastruktur mit ihrer räumlichen Verbreitung nur ein erklärender Faktor ist, der nur so weit wie nötig hinzugezogen wird. So kann die Behauptung formuliert werden, daß die sozialgeographischen Arbeiten keine Erklärung der Standorte der Infrastruktur intendieren; allenfalls partiell liefert die Nutzung der Infrastruktur Erkenntnisse über ihre Verbreitung.

Da sich die Forschungsarbeiten der Sozialgeographie auf das Verhalten menschlicher Individuen im Raum konzentrieren, finden vor allem die konsumnahen Infrastrukturbereiche starke Beachtung, also die Infrastrukturbereiche, die primär und unmittelbar der Versorgung der Bevölkerung dienen. Daher kann als eine weitere Behauptung formuliert werden, daß die für Unternehmen direkt wirksame Infrastruktur in der sozialgeographischen Forschung stark in den Hintergrund tritt, während die indirekt wirksame, konsumnahe Infrastruktur im Mittelpunkt des Interesses steht.

Im folgenden soll in verschiedenen Bereichen der materiellen Infrastruktur geprüft werden, inwieweit sich die Geographie mit der Standortproblematik der Infrastruktur befaßt hat. Dabei soll auch kurz berührt werden, welchen Anteil die Geographie an der standorttheoretischen Forschung des jeweiligen Infrastrukturbereichs hat. Verschiedene Bereiche können wegen des begrenzten Umfangs dieses Aufsatzes nur kurz oder gar nicht erwähnt werden wie etwa die Infrastrukturbereiche "Wohnen» und «Kultur», deren Einrichtungen auch nur von einem Teil der Autoren als Infrastruktur bezeichnet wer- 
den. Lediglich der Bereich von Fremdenverkehr und Freizeit wird exemplarisch etwas weiter ausgeführt.

\subsection{Geographische Arbeiten zur Infrastruktur allgemein}

Geographische Arbeiten, in denen die Verbreitung von Einrichtungen verschiedener Infrastrukturarten in einem Untersuchungsgebiet dargestellt ist, sind zahlreich. Sie können ihren Zweck als planerische Grundlage erfüllen, dienen aber nur selten dem Gewinnen neuer Erkenntnisse für die Theoriebildung innerhalb der Geographie.

Stärker theoriebetont ist eine empirische Analyse der Infrastruktur im Landkreis Miesbach von GRÄF (1978). Neben der Entwicklung der Infrastruktur werden Angebotsmuster und Reichweite, Ausstattungstypen und besonders gruppenspezifische Nachfragemuster der konsumnahen Infrastruktur sowie entsprechende Zusammenhänge herausgearbeitet. Damit wird diese sozialgeographische Arbeit sowohl dem standorttheoretischen als auch dem sozialgeographischen Ansatz gerecht.

\subsection{Geographische Arbeiten zum industriellen Bereich}

Der industrielle Bereich gliedert sich scharf in zwei Forschungsrichtungen:

- Einerseits werden in zahlreichen Arbeiten der Industrie-Geographie die Standortfaktoren verschiedener Branchen oder die Industriestruktur bestimmter Gebiete behandelt. Die Theorie der Standortwahl wird freilich beherrscht von wirtschaftswissenschaftlichen Arbeiten.

- Andererseits werden die Pendlereinzugsbereiche von Betrieben, das Pendelverhalten der Erwerbstätigen oder die Wanderungsmotive der Bevölkerung analysiert, Gebieten, die von ihrer Thematik her der Sozialgeographie entgegenkommen und in denen die Geographie auch Maßgebliches zur Theoriebildung beiträgt.

Wie die Arbeit von THÜRAUF (1975) zeigt, lassen sich der standorttheoretische und der sozialgeographische Ansatz kaum sinnvoll miteinander verknüpfen, da die Bevölkerung eines bestimmten Gebietes kaum Einfluß auf die Standortwahl der Unternehmer hat. Besonderes Interesse verdienen die Auswirkungen der wirtschaftsnahen Infrastruktur, wie sie im Rahmen der Gemeinschaftsaufgabe «Verbesserung der regionalen Wirtschaftsstruktur» gefördert wird. Allerdings wird die Standortwahl der Unternehmer auch durch die konsumnahe sowie die administrative und kommunikative Infrastruktur beeinflußt.

Ausgesprochene Erfolgskontrollen und Wirkungsanalysen zur Industrieansiedlung liegen fast nur von wirtschaftswissenschaftlicher Seite vor. SCHLIEBE (z. B. 1979) berichtet über die Standorte der geför- derten Industriebetriebe sowie über die Motive der Unternehmer.

\subsection{Geographische Arbeiten zu Fremdenverkehr und Freizeit}

Mit diesem Infrastrukturbereich haben sich inzwischen zahllose geographische Arbeiten beschäftigt. Es wurde hauptsächlich die Sozialstruktur der Nutzer untersucht, wobei die vorhandene Infrastruktur in der Regel nur randlich erwähnt, gelegentlich kartographisch dargestellt wird. Gegenüber der Fülle von Arbeiten, die den sozialgeographischen Ansatz verfolgen, haben standorttheoretische Analysen von Geographen Seltenheitswert.

KLÖPPER (1972) hat die gesamte Fremdenverkehrsinfrastruktur (Betriebe und öffentliche Einrichtungen) in der Bundesrepublik analysiert und in verschiedener Hinsicht typisiert; dabei blieben räumliche Aspekte weitgehend unberücksichtigt. Bei den neuen Feriengroßprojekten außerhalb des Zonenrandgebietes haben BECKER et al. (1979) ihre Verbreitung dargestellt und aus den Motiven für die Standortwahl erklärt.

PAECH/RASE (1977) haben bei vier Freizeiteinrichtungen die bundesweite Verbreitung dargestellt und den Versorgungsgrad der Landkreise berechnet.

In mehreren Studien wurden standorttheoretischer und sozialgeographischer Ansatz verknüpft. Zur Abgrenzung des Freizeitraumes Oberstaufen haben MAIER/RUPPERT (1976) die Fremdenverkehrsinfrastruktur kartiert, ihre Verteilung erklärt und bei mehreren Freizeiteinrichtungen die Herkunft und Sozialstruktur der Nutzer erfaßt. KERSTIENS-KOEBERLE (1975) hat in Teilgebieten Oberbayerns die Freizeitinfrastruktur ermittelt, zum Teil kartographisch dargestellt und Dispersionsfaktoren für mehrere Gebiete berechnet und interpretiert. PHILIPP (1975) hat die Verbreitung der Seilbahnen und Lifte in den Bayerischen Alpen kartiert, erklärt und ihre Nutzerstruktur untersucht. Gleiches unternahm HEINRITZ (1976) für die Wildparks und Märchenwälder in Bayern. Besonders hervorzuheben ist eine Analyse der städtischen Freizeitinfrastruktur: In München hat KERSTIENS-KOEBERLE (1979) verschiedene Freizeiteinrichtungen kartiert, ihre Verbreitung - auch in ihrer zeitlichen Entwicklung - erklärt und in einigen Stadtvierteln das Freizeitverhalten der Bevölkerung erfaßt. Schließlich hat DEGENHARDT (1977) die Verbreitung von 12 Freizeiteinrichtungen ermittelt, um die günstigen Wohn- und Lebensbedingungen im ländlichen Raum herauszuarbeiten.

Bei diesen gar nicht sehr zahlreichen standorttheoretischen Untersuchungen erfolgt die Theoriebildung allgemein durch eine generalisierende, erklärende Interpretation der Verbreitungsmuster, teilweise auch durch Analyse von errechneten Dichtewerten. Einige Ansätze zu einer Erfolgskontrolle für die öf- 
fentlichen Fördermittel wurden unternommen. Die Erfahrungen von BECKER(1975) und KLÖPPER(1976) zeigen, daß nur ein schwacher Zusammenhang zwischen dem Ausbau der Fremdenverkehrsinfrastruktur und der Fremdenverkehrsentwicklung besteht. Sinnvoller ist eine Wirkungsanalyse, die zahlreiche positive und negative Aspekte berücksichtigt, wie bei BECKER/KLEMM (1978) und UTHOFF (1976). MAIER/RUPPERT (1974) geben einen Erfolgsbericht über Naherholungsprojekte und ihre Nutzung.

Im Rahmen eines nutzwertanalytischen Ansatzes wurden verschiedene Verfahren zur Bewertung der Landschaft für die Erholung entwickelt. In diesem Rahmen wurde teilweise auch die Freizeitinfrastruktur bewertet. Allerdings ist gerade die Planungstauglichkeit derartiger zusammenfassender Werte für die Freizeitinfrastruktur umstritten (BECKER 1980, 197 ff.).

Insgesamt haben sich Geographen im Sektor Fremdenverkehr und Freizeit maßgeblich beteiligt. Vor allem bei der Verbreitung und Standorterklärung, bei der Analyse der Nutzerstruktur sowie bei der Erfolgskontrolle für die Fremdenverkehrs- und Freizeitinfrastruktur dominieren geographische Beiträge gegenüber den Arbeiten von Nachbardisziplinen. Nur bei den Bewertungsverfahren stehen geographische Arbeiten mehr im Hintergrund. Eine wesentliche Ursache für das starke Engagement der Geographie im Bereich der Fremdenverkehrs- und Freizeitinfrastruktur dürfte die Konsumentennähe und die Beliebigkeit der Inanspruchnahme dieses Infrastrukturbereiches sein, die ihn als Untersuchungsobjekt für die Sozialgeographie besonders attraktiv macht.

\subsection{Geographische Arbeiten zum Bildungssektor}

Unter den verschiedenen Einrichtungen der Bildungsinfrastruktur wurden vor allem Gymnasien und Universitäten von Geographen untersucht. Insbesondere GEIPEL hat in zahlreichen Arbeiten die Entwicklung der Standorte, ihre Nutzung nach sozialen Gruppen und Herkunftsgebieten sowie ihre regionalpolitischen Effekte analysiert. Schon frühzeitig führte er Wirkungsanalysen durch, in denen besonders die Auswirkungen neuer Einrichtungen auf das Bildungsverhalten dargestellt werden. Mit Hochschulstandorten haben sich BAHRENBERG (1974) und MAYR (1970) befaßt sowie MONHEIM (1977) mit den raumordnungspolitischen Effekten von Hochschulen. BAHRENBERG (1976) entwickelt ein Modell zur Bestimmung von Kindergartenstandorten. Trotz dieser maßgeblichen Beiträge von Seiten der Geographie dominieren bei der Bildungsinfrastruktur Arbeiten von Pädagogen.

\subsection{Geographische Arbeiten zur Verkehrsinfrastruktur}

Allein mit den Standorten des Verkehrs oder mit Verkehrsnetzen befassen sich zahlreiche kleinere geographische Arbeiten. Recht abstrakte netztheoretische Studien zu Eisenbahnnetzen oder zum Telefonnetz wurden von mehreren Autoren angefertigt. SCHLIEPHAKE (1973) analysiert in Mittelhessen die Verkehrsinfrastruktur, stellt ihre raumbedeutsamen Auswirkungen dar und befaßt sich vor allem mit dem Erschließungseffekt der Verkehrsinfrastruktur. MAIER (1976) beschreibt die Verkehrsinfrastruktur in Südbayern, um dann - entsprechend dem sozialgeographischen Ansatz - die Verkehrsaktivitätenmuster zur Befriedigung der einzelnen Daseinsgrundfunktionen nach sozialen Gruppen und Reichweiten zu analysieren. In mehreren, meist kleineren Arbeiten werden die regionalpolitischen Effekte neuer Autobahnen oder Flußkanalisierungen herausgearbeitet. Dennoch ist unübersehbar, daß sich die Geographie nur randlich mit der Verkehrsinfrastrukturbefaßt. DieserForschungsbereich wird heute hauptsächlich von der Verkehrswissenschaft getragen, die sich aus den Wirtschaftswissenschaften entwickelt hat.

\subsection{Geographische Arbeiten zur Energie-, Wasser- und Gesundheitsversorgung}

Die Infrastruktur zur Energie-, Wasser- und Gesundheitsversorgung ist von geographischer Seite bislang fast nur marginal behandelt worden. Ursache dieses Defizits dürfte sein, daß einerseits etablierte Fachwissenschaften auf diesen Forschungsfeldern bestehen und daß andererseits diese Gebiete - mit Ausnahme des Gesundheitswesens - für den sozialgeographischen Ansatz weniger interessant sind, da sich keine sozialgruppenspezifische Nutzung ermitteln läßt. Im Rahmen des Gesundheitswesens wurde 1977 eine Untersuchung über die räumliche Verteilung von Ärzte-Niederlassungen veröffentlicht (WULF). Zu erwähnen ist ferner eine rein theoretische Studie über die technische Infrastruktur von BECKER/WENDT (1977) sowie eine Analyse der Energiewirtschaft im Bundesgebiet von MAYER (1974).

\subsection{Geographische Arbeiten zur Zentralität}

Zur Einkaufsinfrastruktur der verschiedenen Zentrenarten, zu ihren Einzugsbereichen und zur gruppenspezifischen Nutzung gibt es zahlreiche geographische Arbeiten. Besonders wurden auch neue Einkaufszentren und Supermärkte untersucht. Dennoch dominieren in diesem Bereich wirtschaftswissenschaftliche Arbeiten, da das Marketing und die Standortwahl der Einzelhandelsbetriebe ganz entscheidende Bedeutung für deren wirtschaftlichen Erfolg haben. 
Die Einkaufsstruktur bildet einen wesentlichen Teil der Zentralität der Städte, es gehört aber auch das Bildungs- und Gesundheitswesen sowie die öffentliche Verwaltung dazu. Diese Zentralität von Gemeinden hat als Basis - neben einer entsprechenden Nachfrage - ein Bündel verschiedenster Infrastruktureinrichtungen. Diese Bündelung der wirtschaftsund konsumnahen Infrastruktur verschafft den zentralen Orten bedeutende Agglomerationsvorteile, die sowohl gewerbliche Betriebe anziehen als auch Haushalte die Nähe zum zentralen Ort suchen lassen. Gerade eine zusammenfassende Betrachtung dieser Infrastruktur und sogar die Bildung räumlicher Modelle hat sich zu einem traditionellen Forschungszweig der Anthropogeographie entwickelt.

Das Modell von CHRISTALLER wurde vielfach angewendet und weiterentwickelt. Eine aktuelle Übersicht bietet HEINRITZ (1979). Das Maß der Realitätserklärung und die Möglichkeiten einer Dynamisierung der zentralörtlichen Theorie hat erst jüngst DEITERS (1978) erörtert. Sowohl die Verbreitung der zentralen Orte als auch ihre gruppenspezifische Inanspruchnahme wurden vielfach untersucht und in der Regional- und Landesplanung verwendet. In der Regional- und Landesplanung bildet das ZentraleOrte-System das dominierende Planungsinstrument, an dem sich der Ausbau der Infrastruktur orientieren soll. Auch die Förderung der Industrieansiedlung stützt sich auf das Zentrale-Orte-System. In der Zentralitätsforschung dominieren Arbeiten von Geographen.

\section{Zusammenfassung und Ausblick}

Die Theorie der Infrastruktur ist eine Domäne der Wirtschaftswissenschaften, die freilich die Lokalisation der Infrastruktur meist nur randlich oder gar nicht beachten. Durch die Angewandte Geographie wurde der Begriff der Infrastruktur in die Geographie getragen, wo er noch keinen festen Platz hat. Dennoch hat sich die Anthropogeographie intensiv mit der Infrastruktur befaßt. Im Rahmen des sozialgeographischen Ansatzes wurde und wird insbesondere die gruppenspezifische Nutzung der Infrastruktur analysiert. Durch ihn wurde der standorttheoretische Ansatz in den Hintergrund gedrängt:

- In den sozialgeographischen Arbeiten wird die Verbreitung der Infrastruktur allgemein nur so weit wie nötig erklärt, während der Schwerpunkt in der Regel auf der gruppenspezifischen Nutzung liegt.

- Durch das Interesse an den Nutzern hat sich die Sozialgeographie auf die konsumnahe Infrastruktur konzentriert, bei der allein das Gesundheitswesen fast unberücksichtigt blieb. Im Rahmen der Industrie-undFremdenverkehrsgeographiewurde auch die wirtschaftsnahe Infrastruktur analysiert.
Andere Forschungszweige haben sich stark bei der Infrastrukturforschung engagiert. Maßgebliches zur Theoriebildung hat die Anthropogeographie nicht nur bei der Nutzung der konsumnahen Infrastruktur beigetragen, sondern auch beim Arbeitspendeln, bei der Standorttheorie von Fremdenverkehr und Freizeit sowie besonders bei der Zentralitätsforschung. Dagegen hat die Geographie im Vergleich mit anderen Wissenschaften nur wenig an Erkenntnissen zur Infrastruktur des Verkehrs, des Gesundheitswesens und der Energie- und Wasserversorgung geliefert.

Für die künftige geographische Infrastrukturforschung ist anzustreben, daß der standorttheoretische und der sozialgeographische Ansatz miteinander verknüpft werden, indem die Lokalisation der Infrastruktur im Zusammenhang mit der Nutzerstruktur analysiert wird. Vor allem durch derartige Wirkungsanalysen können wichtige Entscheidungsgrundlagen für die Planung erarbeitet werden, insbesondere hinsichtlich der räumlichen Voraussetzungen von Infrastrukturinvestitionen sowie deren Auswirkungen. Damit könnte die Planungstauglichkeit der geographischen Forschung wesentlich verbessert werden.

In diesem Rahmen könnte und sollte die Geographie auch Grundlagen für die Lösung einiger aktueller planerischer Probleme liefern:

- Kann angesichts des Bevölkerungsrückganges eine angemessene infrastrukturelle Versorgung des ländlichen Raumes durch neue räumliche Organisationsformen sichergestellt werden?

- Welche Probleme ergeben sich aus der gegenwärtigen Tendenz, die höherrangigen Zentren nicht mehr besonders zu stärken und stattdessen die Infrastruktur in den Unter- und Mittelzentren besonders zu fördern?

- Kann die mobile Infrastruktur, die zunehmend Dienstleistungen im ländlichen Raum erbringt, wesentliche Aufgaben zur Versorgung dieses Raumes erfüllen?

- Mit welchen Auswirkungen muß die bestehende Infrastruktur rechnen, wenn neue Technologien wie etwa der Bildschirmtext in der Telekommunikation - sich stärker durchsetzen?

- Welche Auswirkungen verursacht die Zersiedlung in den Randbereichen der Verdichtungsräume, vor allem, wenn dort die Infrastruktur immer erst hinterhergetragen wird?

\section{Literatur}

BAHRENBERG, G.: Zur Frage optimaler Standorte von Gesamthochschulen in Nordrhein-Westfalen. Eine Lösung mit Hilfe der linearen Programmierung. In: Erdkunde, 28., 1974, S. 101-114.

BAHRENBERG, G.: Ein sozial gerechtes Optimierungsmodell für die Standortwahl von öffentlichen Ein- 
richtungen. In: Verhandlungen des Deutschen Geographentages, 40., 1976, S. 443-454.

BECKER, CHRISTOPH: Raumbedeutsame Ausgaben in Gebieten des Erholungsreiseverkehrs - eine Untersuchung zur Problematik raumbedeutsamer Ausgaben in verschiedenen Funktionsräumen. In: Räumliche Wirkungen öffentlicher Ausgaben, Veröffentlichungen der Akademie für Raumforschung und Landesplanung, Forschungs- und Sitzungsberichte, 98., Hannover 1975, S. 131-212.

BECKER, CHRISTOPH und KLEMM, KR.: Raumwirksame Instrumente des Bundes im Bereich der Freizeit. Schriftenreihe des Bundesministers für Raumordnung, Bauwesen und Städtebau, 06.028, Bonn 1978. BECKER, CHRISTOPH et al.: Feriengroßprojekte außerhalb des Zonenrandgebietes - Struktur und regionalpolitische Effekte. - Materialien zur Fremdenverkehrsgeographie, 4., Trier 1979.

BECKER, CHRISTOPH: Die Anwendung verschiedener Landschaftsbewertungsverfahren auf sechs deutsche Fremdenverkehrsgebiete - ein Vergleich. In: Empirische Untersuchungen zur äußeren Abgrenzung und inneren Strukturierung von Freizeiträumen, Veröffentlichungen der Akademie für Raumforschung und Landesplanung, Forschungs- und Sitzungsberichte, 132., Hannover 1980, S. 159-205.

BECKER, CHRISTIANE und WENDT, H.: Die technische Infrastruktur - ihre Bedeutung als Standortfaktor. In: Petermanns Geogr. Mitteilungen, 121., 1977, S. 27-35.

BLOTEVOGEL, H.H. und HEINEBERG, H.: Bibliographie zum Geographiestudium. Teil 2. Paderborn 1976.

BOESCH, H.: Weltwirtschaftsgeographie. (4. Aufl.) Braunschweig 1977.

BOESLER, K.A.: Wandlungen in der räumlichen Struktur der Standortqualitäten durch die öffentlichen Finanzen im Nordteil des Modellgebietes. In: Die Ansprüche der modernen Industriegesellschaft an den Raum. 2. Teil. Veröffentlichungen der Akademie für Raumforschung und Landesplanung, Forschungs- und Sitzungsberichte, 74., Hannover 1972, S. 31-80.

TEN BRINK, M. und GATZWEILER, H.P.: Erfolgskontrolle raumwirksamer Mittel als Voraussetzung für eine integrierte Raumentwicklungsplanung. In: Raumforschung und Raumordnung, 34., 1976, S. 256-269.

BUHR, W.: Die Rolle der materiellen Infrastruktur im regionalen Wirtschaftswachstum. Berlin 1975.

DEGENHARDT, B.: Das Freizeitpotential des ländlichen Raumes. In: Neues Archiv für Niedersachsen, 26., 1977, S. 23-47.

DEITERS, J.: Zur empirischen Überprüfbarkeit der Theorie zentraler Orte. Arbeiten zur Rheinischen Landeskunde, 44., Bonn 1978.
FREY, R. L.: Infrastruktur. Grundlagen der Planung öffentlicher Investitionen. 2. Aufl. Tübingen/Zürich 1972.

GRÄF, P.: Zur Raumrelevanz infrastruktureller Maßnahmen. Kleinräumliche Struktur- und Prozeßanalyse im Landkreis Miesbach, ein Beitrag zur sozialgeographischen Infrastrukturforschung. In: Münchner Studien zur Sozial- und Wirtschaftsgeographie, 18., Kallmünz/Regensburg 1978.

HANSER, CHR. et al.: Auswirkungen von Infrastrukturinvestitionen. 1. Wissensch. Zwischenbericht: Methodische Vorstudie. Zürich 1979.

HARD, G.: Die Geographie. Eine wissenschaftstheoretische Einführung. Berlin/New York 1973.

HEINRITZ, G.: Wildparke und Märchenwälder. Zur Ausrüstung und Differenzierung neuer Freizeitparks in Bayern. In: Natur und Landschaft, 51., 1976, S. 15-19.

HEINRITZ, G.: Zentralität und zentrale Orte. Stuttgart 1979.

ISTEL, W.: Voraussetzungen und Möglichkeiten zur Durchführung der Infrastruktur-Investitionsplanung. München 1976.

JANSEN, P.G.: Infrastrukturinvestitionen als Mittel der Regionalpolitik. Beiträge zur Raumplanung, 3., Gütersloh 1968.

JOCHIMSEN, R.: Theorie der Infrastruktur. Tübingen 1966.

KERSTIENS-KOEBERLE, E.: Raummuster und Reichweiten der freizeitorientierten Infrastruktur. In: Geographische Rundschau, 27., 1975, S. 18-30.

KERSTIENS-KOEBERLE, E.: Freizeitverhalten im Wohnumfeld. Münchner Studien zur Sozial- und Wirtschaftsgeographie, 19., Kallmünz/Regensburg 1979.

KLÖPPER, R.: Struktur- und Ausstattungsbedarf in Erholungsorten der BRD. Hannover 1972.

KLÖPPER, R.: Änderung der Angebots- und Nachfragestrukturen im Fremdenverkehr in ausgewählten Regionen. Hannover 1976.

KUHN, A.: Erarbeitung von praktisch anwendbaren Grundlagen und Methoden für die Koordinierung des Einsatzes raumwirksamer Bundesmittel. Hannover 1972.

MAIER, J.: Zur Geographie verkehrsräumlicher Aktivitäten. Münchner Studien zur Sozial- und Wirtschaftsgeographie, 17., Kallmünz/Regensburg 1976.

MAIER, J. und RUPPERT, K.: Geographische Aspekte kommunaler Initiativen im Freizeitraum. Münchner Studien zur Sozial- und Wirtschaftsgeographie, 9., Kallmünz/Regensburg 1974.

MAIER, J. und RUPPERT, K.: Freizeitraum Oberstaufen. WGI-Berichte zur Regionalforschung, 13., München 1976. 
MAYER, F.: Die Energiewirtschaft der Bundesrepublik Deutschland. Gegenwartsanalyse und $\mathrm{Zu}$ kunftsperspektiven. In: Geographische Rundschau, 26., 1974, S. 257-273.

MAYR, A.: Standort und Einzugsbereich von Hochschulen. In: Berichte zur deutschen Landeskunde, 44., 1970, S. 83-110.

MONHEIM, H.: Die raumordnungspolitische Relevanz des Hochschulbaus. In: Informationen zur Raumentwicklung, 1977, S. 201-219.

PAECH, E.-M. und RASE, W.-D.: Versorgungssituation der Kreise mit Basiseinrichtungen für Freizeitsport. In: Raumforschung und Raumordnung, 35., 1977, S. 63-68.

OTREMBA, E.: Allgemeine Agrar- und Industriegeographie. (2. Aufl.) Stuttgart 1960.

PHILIPP, W.: Seilbahnen und Lifte im bayerischen Alpenraum. Wirtschaftsgeographische Aspekte freizeitorientierter Infrastruktur. WGI-Berichte zur Regionalforschung, 13., 1976.

Raumordnungsbericht 1968 der Bundesregierung. Verhandlungen des Deutschen Bundestages. Drucksache V/3958 vom 12.3.1969. (Bonn 1969.)

\section{Literaturbesprechnung}

BOWLER, I.R.: Government and Agriculture: A Spatial Perspective. London: Longman Group Ltd., 1979.127 S., £ 4.50. (ISBN 0-582-48578-9)

Das Buch wendet sich vor allem an Studierende der Geographie, aber auch der Nachbárdisziplinen. Vf. geht aus von dem Faktum, dass die Gesetzgebung der Regierungen bislang von Geographen bezüglich ihrer Raumwirksamkeit kaum untersucht worden ist. Agrarpolitische Entscheidungen sind für eine solche Analyse besonders geeignet, weil sie sich an eine klar umrissende Zielgruppe richten und zumeist flächenhafte Wirkungen zeigen.

Der 1. Teil des Buches (Kap. 1-3) bringt eine Literaturübersicht, wobei vorwiegend Arbeiten der Politik- und Wirtschaftswissenschaft, Soziologie und Geographie berücksichtigt werden. Die einzelnen Kapitel beschäftigen sich mit: (1) Agrarpolitische Ziele und politische Prozesse, (2) Agrarwirtschaftliche Probleme und politische Massnahmen, (3) Bewertung der Agrarpolitik. Der 2. Teil (Kap. 4-7) wendet sich den spezifischen Problemen der agrarpolitischen Gesetzgebung in Grossbritannien im Zeitraum zwischen 1900 und $1973 \mathrm{zu}$. Insbesondere die Fallstudien in den Kap. 6 und 7 kommen den Zielsetzungen des Autors wohl am nächsten. Hier geliegt es ihm, die Raumwirksamkeit der Massnahmen, ihre Reichweite und ihre raum-zeitliche Diffusion zu erfassen. Die Darstellung wird durch zahlreiche Abbildungen und Tabellen sinnvoll ergänzt.

Abschliessend werden die Ergebnisse der Untersuchung zusammengefasst.
REUTER, W.-H.: Zur Problematik raumwirksamer Finanzen im Kommunal-Bereich am Beispiel des Landkreises Bersenbrück. Bielefeld 1974.

SCHÄTZL, L.: Wirtschaftsgeographie 1. Theorie. Paderborn 1978.

SCHLIEBE, K.: Zum Standortverhalten der Industriebetriebe. Informationen zur Raumentwicklung, 1979, S. 351-362.

SCHLIEPHAKE, K.: Geographische Erfassung des Verkehrs. Gießener Geographische Schriften, 28., Gießen 1973.

THURAUF, G.: Industriestandorte in der Region München. Münchner Studien zur Sozial- und Wirtschaftsgeographie, 16., Kallmünz/Regensburg 1975.

UTHOFF, D.: Analyse räumlicher und regionalwirtschaftlicher Auswirkungen staatlich geförderter $\mathrm{Fe}$ rienzentren. Hannover 1976.

VOPPEL, G.: Wirtschaftsgeographie. Stuttgart 1970. WULF, K.: Die räumliche Verteilung der Ärzte-Niederlassungen in Schleswig-Holstein und ihre Dynamik 1969-1976. MARE-Materialien zur Geogr. Regionalforschung in Kiel, 1., Kiel 1977.
Die wichtigsten Aussagen sind:

- Aufgrund fehlender Studien lassen sich kaum generelle Aussagen zur Raumwirksamkeit der Agrarpolitik treffen,

- die vorliegenden Ergebnisse haben zumeist nur Gültigkeit für einen speziellen Fall und eine bestimmte Region,

- Bewertungskriterien für agrarpolitische Entscheidungen sind bislang fast nur von Wirtschaftswissenschaftlern vorgelegt worden, - es konnte auf der Grundlage der Fallstudien in Grossbritannien kein überzeugender Beweis dafür gefunden werden, dass die Gesetze agrarstrukturelle Wandlungsprozesse einleiten. Sie hatten zwar eine verstärkende Wirkung, doch kann davon ausgegangen werden, dass die Strukturwandlungen auch allein durch die sozialen und ökonomischen Rahmenbedingungen hervorgerufen worden wären.

Es ist dem Vf. zu bescheinigen, dass er mit seinem Buch den Anstoss gegeben hat zu einer genaueren Beschäftigung mit der Raumwirksamkeit des Gesetzgebers, allerdings ist die Darstellung in der vorliegenden Form noch zu wenig "geographisch". Die Fallstudien im 2. Teil zeigen auf, in welche Richtung zukünftige Forschungsvorhaben gehen sollten. Bedauerlich ist, dass der Vf. sich bei seiner Literaturauswertung, wie leider bei jüngeren Publikationen aus dem englischen Sprachraum häufig festzustellen, fast ausschliesslich auf englischsprachige Arbeiten bezieht. Hans-IVilhelm Windhorst Osnabrück/Vechta 